\title{
MangaSticker: A Tool to Build Interactive Manga
}

\author{
Vitor de Almeida Silva \\ Pontifical Catholic University of \\ Goiás, PUC Goiás Goiânia/GO \\ Brazil
}

\author{
Lutyéllen dos Santos Ribeiro \\ Pontifical Catholic University of \\ Goiás, PUC Goiás Goiânia/GO \\ Brazil
}

\author{
Talles Marcelo Gonçalves de \\ Andrade Barbosa \\ Pontifical Catholic University of \\ Goiás, Goiânia/GO Brazil
}

\begin{abstract}
Circuit stickers are circuits embedded in stickers that allow interactive devices using everyday materials, such as paper. The educational manga uses manga's characteristics, such as big eyes, large heads, and detailed scenes, to teach some specific concept in a simplified and fun way. This paper proposes a systems software called MangaSticker, which integrates the circuit sticker with the educational manga to build interactive mangas. Three test cases were presented and implemented to evaluate the usability of MangaSticker. Such test cases have been customized to support money handling skills training activities for people with Down Syndrome (DS).
\end{abstract}

\section{General Terms}

Processing IDE, Java, Manga, Circuit sticker, ControlP5, Firmata

\section{Keywords}

Interactive manga, Down Syndrome, Handle Money Skills, Circuit sticker, Paper circuit, Educational manga, Tangible interfaces

\section{INTRODUCTION}

Circuit stickers are modules of flexible circuit components with a conductive adhesive base. The circuit sticker allows interactive devices using everyday materials, such as paper [1]. LEDs, buttons, and controllers are used in the construction of these devices [2]. Such artifacts use the concept of tangible interfaces, where the interaction is made through touch. The circuit sticker is commonly used to generate contexts or highlight characters' expressions [2].

Manga is a genre of comic strips originating in Japan that is currently very famous in Western countries. The manga is characterized by scenarios and characters with many details and frames with varied formats. The characters have large eyes and heads and hair with different colors and hairstyles, which are used to highlight the characters' personality and feelings [3]. Manga is available in several categories, such as the youth category (in Japanese - Shounen), comedy category, and educational manga category (In Japanese - Gakushu manga). The youth category produces content without the presence of overly violent scenes. Comedy makes comic content for different ages. The educational manga category uses manga to teach a specific concept in a simplified and fun way [3] [4] [5].

The study's object is creating a tool to integrate the circuit sticker and the educational manga to create interactive manga. For this purpose, MangaSticker was developed, which is an interactive manga modeling environment. This tool makes it possible to print the designs on A4 sheets, facilitating the assembly of the circuit components and using the educational manga superimposed on the circuit sticker.
Three customized test cases for training money handling skills for people with Down Syndrome (DS) were proposed and implemented. Researchers from the Alfadown project maintained by PUC Goiás [6] helped to elicit the requirements for adapting the interactives manga. The integration of controllers was verified through the Firmata communication protocol with the interactive manga. In a complementary way, characters, colors, and formats were evaluated for the population with DS.

\section{BIBLIOGRAPHIC REVIEW}

In this section, the bibliographic review is divided into four topics: Circuit stickers, Educational manga, Teaching people with DS, and Money handling skills for people with DS.

\subsection{Circuit sticker}

Currently, circuit stickers are available in do-it-yourself (DIY) toolkit formats. The PLIX [7] and Chibitronics [8] toolkits combine controller programming tools with circuit stickers. Such toolkits make it possible to create artifacts such as gift cards or interactive drawings.

The "StoryClip" [9] is a circuit sticker toolkit applied in early childhood education. With multimedia resources, the kit makes it possible to record lines for characters drawn freehand on paper. The storyBook "Ellie the LED" [2] developed by MIT researchers, applied a circuit sticker to teach basic concepts, such as constellations. LEDs in various colors and buttons made it possible to highlight the context and emotions of the character. In Santos [10], a smart glove was developed that allows interaction with the circuit sticker. LEDs and sensors were used to create a scenario, which responded with lights to the glove's commands. This tool was evaluated in applications for the literacy of people with DS.

Some works use software systems to create artifacts that make up the circuit sticker in another perspective. "PaperPixels" [11] is a toolkit that allows the creation of animations on paper using a circuit sticker. "I * CATch" [12] is a framework that enables children to develop wearable technologies in the form of circuit stickers. In the "T-books toolkit" [13], an interactive storybook in the form of a circuit sticker was made, allowing changes to the story's narrative. Characters told the stories through audios of lines.

\subsection{Educational manga}

Berndt's work [3] shows that using manga characteristics, such as characters with big eyes and exaggerated expressions, can contribute to activities' attention. It is shown that children's personalities can facilitate the application of activities in the forms of play.

In the Moyashimon-Tales case study [4], the authors show an educational manga applied in agriculture. The character was used as a storyteller, responsible for implementing the activities and keeping readers interested. "Sigma PI" [5] is an educational manga that teaches chemistry concepts. The 
sequence of scenes with reading in the oriental sense, colors in shades of gray, art style shoujo (manga for a female audience), and less formal dialogues, were used in teaching the content.

\subsection{Teaching for individuals with DS}

Usually, there are two different approaches regarding education for individuals with DS. Negative points, which consist of the low development of short-term memory; prearithmetic difficulties; motor problems (picking up, pulling); oro-motor problems (speech); visual problems, and hearing problems (at certain levels). Positives points, which are the excellent development of visual memory; good learning using $2 \mathrm{D}$ or $3 \mathrm{D}$ objects; video/picture modeling learning, and reasonable response to positive reinforcement [14] [15] [16].

\subsection{Money handling skills}

Money handling skills is an area that studies methodologies and applications to teach people how to manage their financial resources [15]. Part of this research seeks to promote autonomy and social integration for individuals with some weakness, whether cognitive or motor [14] [15].

For the training of money handling skills for the population with DS, the work of Maddumarachchi [14] and RUS [15] are highlighted. Maddumarachchi [14] applied the teaching of money handling to the population with DS through video modeling. Video modeling is the teaching of concepts by imitating the behavior of characters in videos. The author applied purchasing activities, realizing that the best way is to divide the main action into smaller and simple steps.

In Rus [15], an interactive table and an application for training money handling skills were created for the DS population. Valuation and purchase activities were tested. The authors highlighted problems with the icons' size, the signs on the notes and coins, and pre-arithmetic skills.

\section{MATERIALS AND METHODS}

At first, the concepts of circuit sticker and educational manga were revised to raise the prototype's requirements. Subsequently, the tool was implemented through successive requirements refinements. The technology used was Processing IDE [17] and the Java programming language, due to the portability, libraries, native primitives of the IDE, and the smaller learning curve.

Two test cases were then developed for the population with DS consisting of two money handling skills activities. Such test cases were produced based on successive refinements of requirements in the interaction with other researchers of the Alfadown project.

\subsection{Implementations of the prototype}

MangaSticker allows the integration between the circuit sticker and the educational manga to build interactive manga. An editing environment was developed to this end, which offers a way to visualize and model the manga and the circuit sticker concurrently.

The development started from an initial requirement, which was the printing of two sheets, where one would be the face of the circuit project and the other the face of the manga. It allows the circuit components to be overlaid with the manga sheet, which results in the interactive manga. It is possible to introduce new figures in the software, leaving the user free to choose between the standard models or create their icons.

Figure 1 shows the class diagram containing the entire organization of the prototype. The graph shows the main classes, such as "Edit_Field," which controls the different layers, "List_Components," used to manage circuit components and figures, and "Persistence," which saves or loads a project.

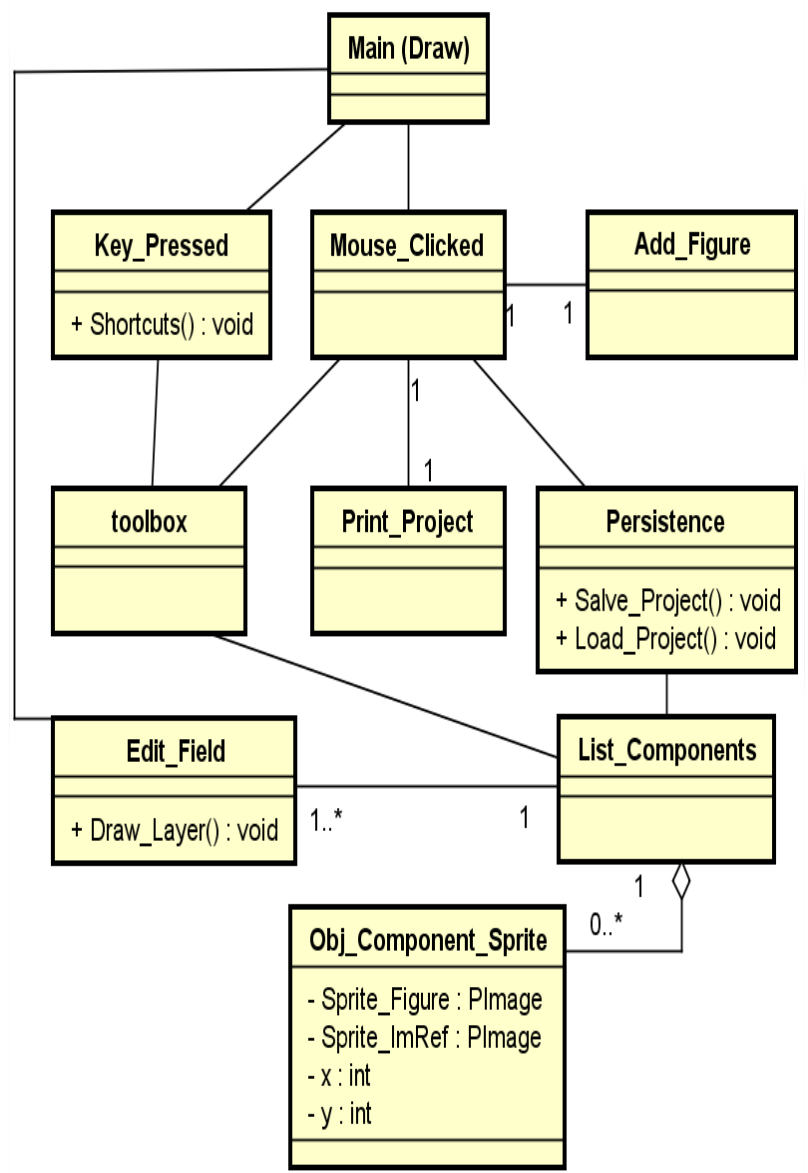

Fig 1: The Structure of the MangaSticker

Figure 2 shows the MangaSticker interface. Such an interface was generated by the refinement and testing of different prototypes. The current layout allows the user to view the tools and the editing field. The following sections show the main aspects of the prototype's implementation based on Figures 1 and 2. 

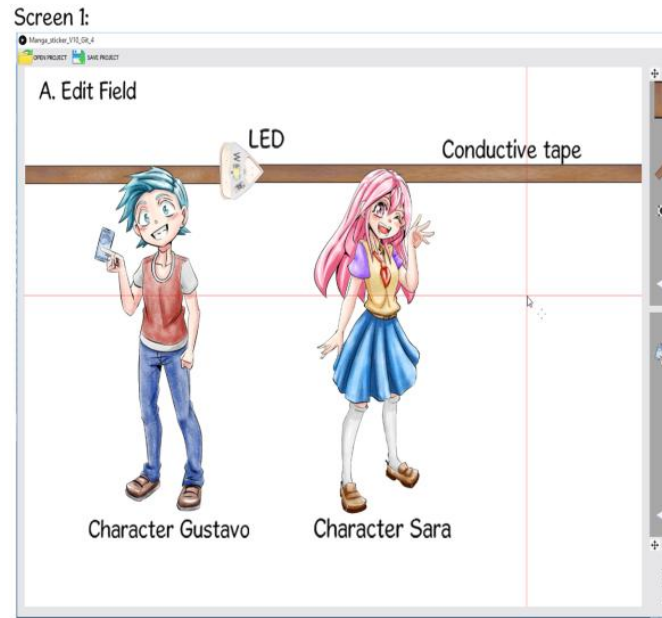

Screen 2:
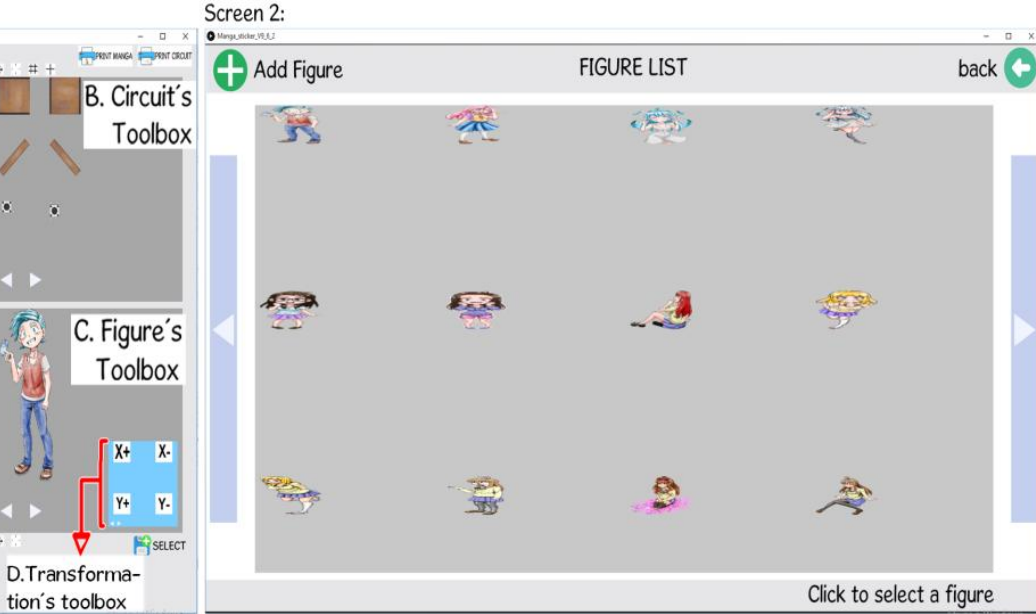

Fig 2: MangaSticker screens, screen 1 (Edition), screen 2 (picture management)

\subsubsection{Layers}

In Processing, there are objects called PGraphics, and such objects allow the creation of layers on the main screen. Layers allow free editing of components without affecting the main program [17]. They were used to separate the fields where the manga is edited and where the circuit is edited. When controlling the layer, it is possible to change the two faces' opacity, allowing the two layers' visualization, which facilitates the items' positioning. The layers also allow printing the circuit sticker and the manga on separate sheets.

\subsubsection{Sprites}

In computer graphics, the sprite is one or more pre-modeled figures that can be manipulated along the interface or animated using several sprites [18]. The sprite concept was combined with Processing's PImage primitive, which provides compatibility with common image extensions like ".jpg" or ".png." The components of figures and circuits were modeled and encapsulated in sprite-shaped objects. The sprite makes it possible to add figures to the environment only, such as edited figures drawn by hand, cell phone, or internet photos.

By default, sprites of characters, pictures, scenarios, animals, and emoticons were inserted in MangaSticker. The user and their sprites can use these standard images or create an entire interactive manga.

\subsubsection{Toolbox}

Toolboxes are a set of features, tools, or options for a program. The toolboxes were developed using the button resources present in the ControlP5 library [19]. ControlP5 is a Processing library that has control functions for the graphical user interface. It is available free of charge under the terms of the GNU (General Public License). The toolboxes were used in the list of components and figures to select the transformations needed. After editions can be applied to the sprites.

\subsubsection{Transformations and tools}

MangaSticker's editing tools were implemented using digital image processing (PDI) techniques [20]. Functions were created for rotation, mirroring, and scale transformations. Rotation functionality moves the sprite image 90 degrees clockwise. Mirroring inverts the sprite image pixels horizontally or vertically. Scale increases the sprite image's width or height by a constant of $0.3 \mathrm{~cm}$ (10 pixels).

The graduated grid and linear XY functions help align the components and visualize the paper's dimensions in units of 1 $\mathrm{cm}$. The drag tool allows moving the sprite around the editing area using the mouse. The select tool makes it possible to choose a specific sprite for editing. The Deleting tool removes sprites from the editing area. The up/down functionality sends down/up, allows overlapping sprites, can be used in positioning backgrounds. The undo and redo provoke the return of actions for the correction of errors or quick changes. The tool for adding pictures allows images to be uploaded from the computer to the MangaSticker. The opacity change tool allows control of the visualization between the two layers. The manga/circuit printing functions generate PDF files of the project ready for printing.

As shown in Figure 2, all options were organized in the form of buttons and toolboxes. They have also been linked to keyboard shortcuts to make the editing process faster and more practical.

\subsubsection{Edit field and print project}

The interactive manga modeling is performed in the editing field, where the user has automatic access to the circuit and manga layers concurrently. The printed project follows the coordinates established in the tool. In this way, the manga sheet becomes the user's interface with the circuit sticker.

The editing area and the printing of the artifacts were made in A4 sheet dimensions. A4 sheets are portable, fit in folders, have space for one or two pages of a manga, and are inexpensive. They are also a typical pattern of sheets that can be easily found in stationery stores.

The screening of 1920x1080 and printing tests was performed to determine the measurements, where it was verified that 1 $\mathrm{cm}$ is equivalent to 47 pixels. An editing area of $27 \mathrm{~cm} \mathrm{x} 19$ $\mathrm{cm}$ was generated, equivalent to an $\mathrm{A} 4$ sheet in a landscape with $2 \mathrm{~cm}$ margins.

\subsubsection{Circuit components}

Based on the MIT researcher QI [2], four basic components were modeled. Conductive tape $(4 \mathrm{~cm} \times 0.9 \mathrm{~cm})$; LEDs $(2 \mathrm{~cm} \mathrm{x}$ $2 \mathrm{~cm})$; push-buttons $(0.5 \mathrm{~cm} \times 0.5 \mathrm{~cm})$ and electrical tape $(4$ $\mathrm{cm} \times 0.9 \mathrm{~cm})$. These components are enough to generate simple models of interactives manga with only buttons and LEDs or more complex models using controllers.

\subsection{Usability tests}

At first, white-box tests were performed on the software to discover errors in logic and coding. Then, black-box tests were performed to calibrate the prints of the designs. Finally, three test cases were implemented for developing money handler skills training. The first activity is for bill 
discrimination training, and the second is for bill price quantification training.

\subsubsection{Customization}

Down syndrome (DS) is a genetic condition that causes physical and cognitive problems for affected individuals [16]. Typically, people with DS have difficulties with short-term memory and learning pre-arithmetic skills. On the other hand, they present good visual memory development and good learning using $2 \mathrm{D} / 3 \mathrm{D}$ characters or objects. Because of this, the Pontifical Catholic University of Goiás (PUC GOIÁS) maintains the AlfaDown project, which seeks the social integration of individuals with DS through the application of computer technology [6].

The process of creating the test cases was accompanied by researchers linked to the Alfadown project. Money training was chosen for the activities, as it can assist in people's autonomous life with DS. Successive refinements of requirements were made to adapt the artifacts to the characteristics of the population.

It was suggested that the activities should be simple and have a visual stimulus, sound stimulus, interactivity, and positive reinforcement, which are recommended requirements for training people with DS. The three most relevant characteristics of interactive manga for this population stand out, the teaching of content through stories; the visual potential (colors, characters, light stimuli); the possibility of placing speech audios for the characters; the ability to interact with the characters and scenes through touch.

Figure 3 shows the flowchart with the sequence of steps of the activities with interactive manga.

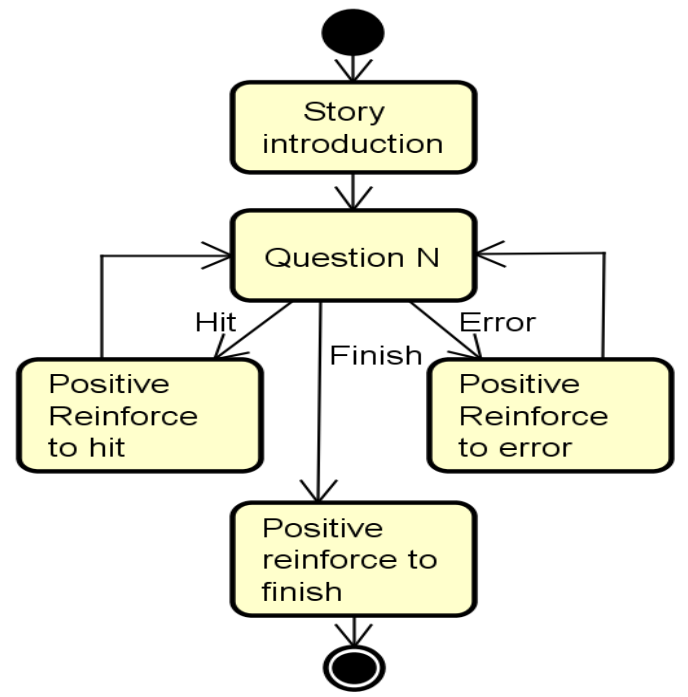

Fig 3: Flowchart for interactive manga activities

The test cases were constructed following the flowchart in Figure 3. At first, a story is introduced, which introduces a character that teaches about the content. Then, there are some questions that the user must answer. For each answer, a type of positive reinforcement is performed for error or success. In the end, positive reinforcement is made for the closure of activities.

Activities with paper money notes were chosen due to their simplicity and because they have individual identification characteristics, which are color and number. Pre-arithmetic skills were avoided to not limit the population to only individuals who have this requirement. Audios and text were used to facilitate the understanding of the content in the characters' speeches. Interactivity was applied in questions and answers to increase attention through the person's involvement with the characters. Congratulations/incentive audios and light stimuli with LEDs were applied to the positive reinforcement. The reading direction chosen was the western one, given that the target audience is already familiar with this reading sense.

\subsubsection{Characters}

Figure 4 shows the character modeling process. It was decided to use two characters, Sara and Gustavo.

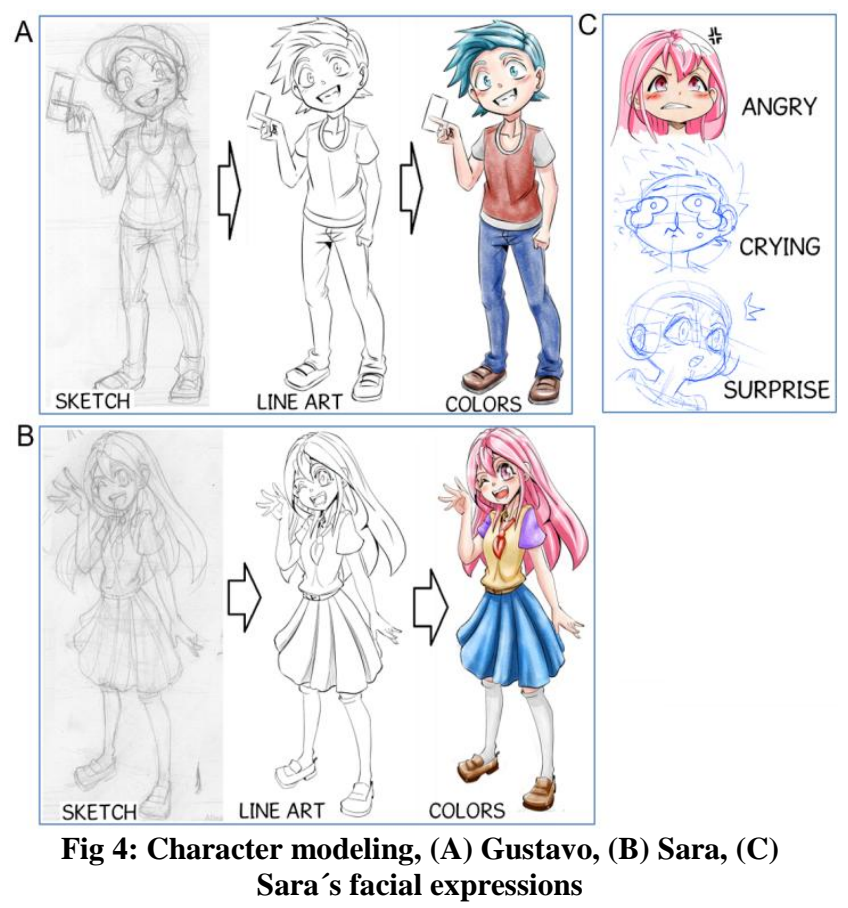

The Chibi style was chosen for the characters. This style is characterized by childish characters with big eyes/heads and exaggerated facial expressions [3]. The colors were selected to be pleasant and inspire a relaxed atmosphere, helping users not be anxious with the characters. The age chosen for the characters was between 11 and 13 years old. Children's preferences facilitate the insertion of contexts where games are played. It can lead individuals to interact better with the characters having fun, identifying themselves, or being captivated by them.

\subsubsection{Test case technology}

The creation of the test cases required the development of hardware and software. MangaSticker was used throughout the process of modeling the circuit sticker and manga. An Arduino Uno with an ATmega328P chip and a 16-channel multiplexer module [21] was used to interface the PC and the interactive manga. Processing IDE and the Firmata library were used to control the manga scenes, reproduce the audios and light stimuli using commands sent to the controller. Firmata is a protocol that allows communication between the computer and a microcontroller through the serial port [22]. It is available free of charge under GNU terms. It was chosen for its ease of use and integrating Arduino programming into Processing, making development faster. 
(a)
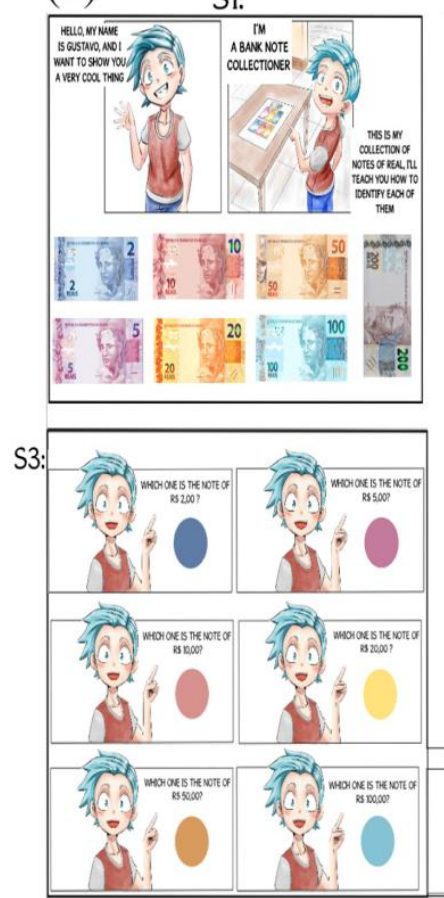

S2:

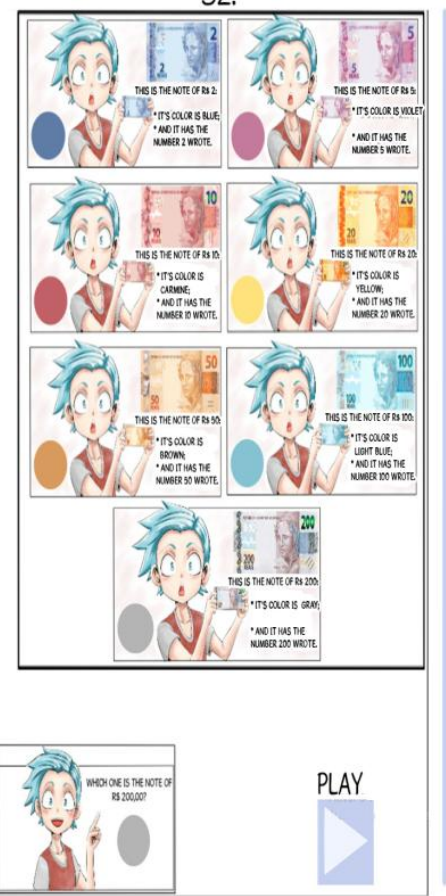

(b)

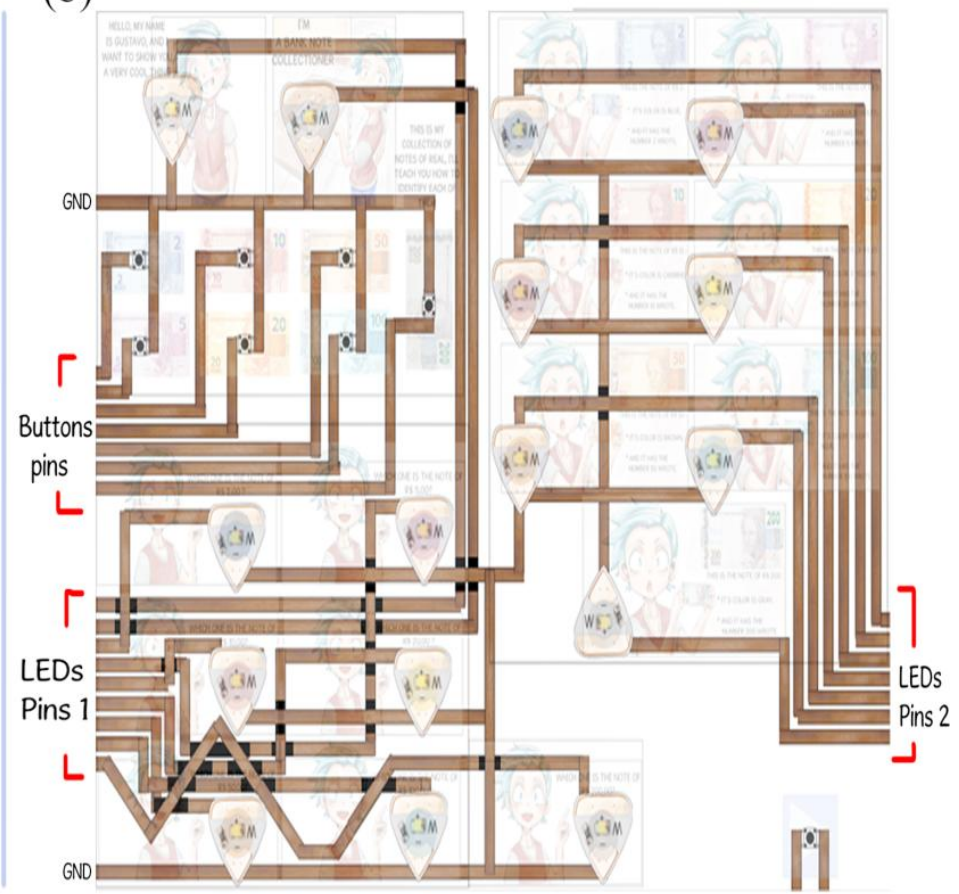

Fig 5: Screenshot MangaSticker, Test case 1: (A) Face of the manga (western reading direction), (B) Circuit sticker (reduced opacity of the manga)

\subsubsection{Test case 1: notes discrimination}

This activity teaches how to identify real notes. The flowchart in Figure 3 was followed, using the character Gustavo. Figure 5 shows the model of test case 1 in the MangaSticker.

The scenes were divided into three frames S1, S2, and S3. The manga tells the story of Gustavo, a collector of paper money. Throughout the novel, Gustavo shows the interlocutor his collection of real notes and tips on identifying each money bill. The directions show that placing the money bill remembering the colors and numbers can facilitate the process. The character's story and concepts were made in frames $\mathrm{S} 1$ and $\mathrm{S} 2$.

In the frame S3, activities are carried out in which Gustavo asks the interlocutor to identify which note is which. The answer is given by pressing the money bill chosen in Gustavo's collection in box S1. As shown in Figure 3, for each response in the activity, positive reinforcements are performed. Audios with clapping and congratulating lines of the character are used in the positive reinforcement of correct answers. In the positive reinforcement of the error, Gustavo encourages the interlocutor to make a new attempt. In the final reinforcement, the character ends the activities and says goodbye, inviting the interlocutor for a try. Figure 6a shows the answer to a question in the model physically implemented. Figure $6 \mathrm{~b}$ shows the LEDs flashing in the positive reinforcement of the hit.

During the process, as mentioned earlier, pictures and scenario items are indicated by the LEDs in conjunction with the character's speech, as shown in Figures 6a and 6b. The circuit sticker is connected to the Arduino that controls the LEDs. The buttons located in frame 1 are the control for the responses of the activities.

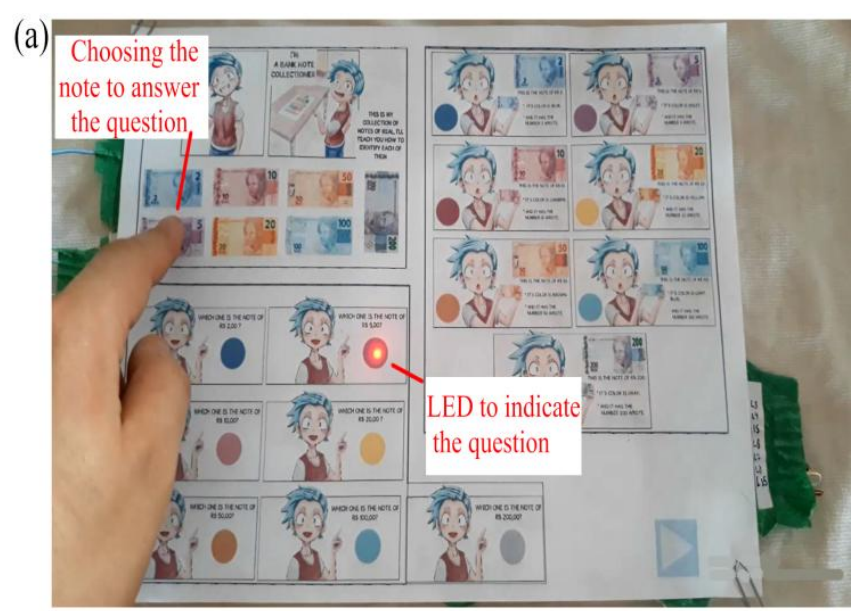

(b)

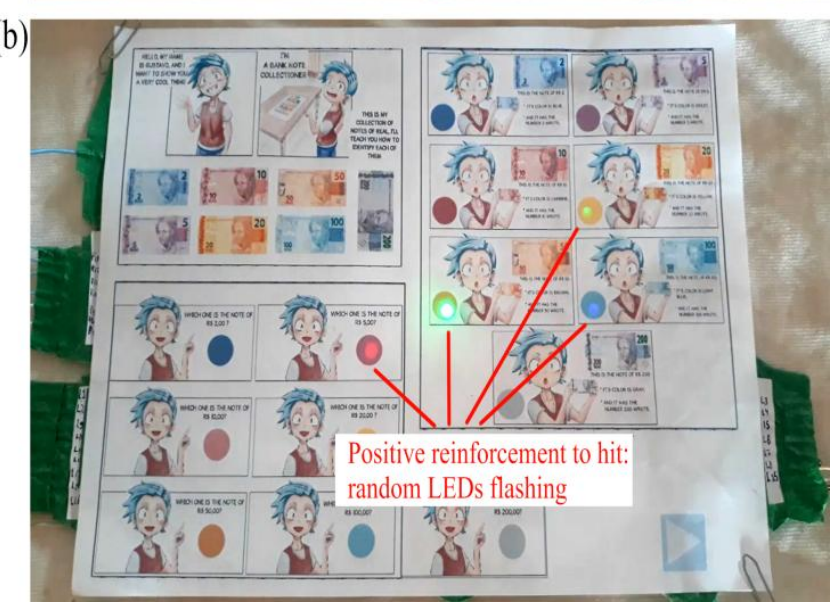

Fig 6: Test case 1 physical model, (a) Answering a question from the character: "which one is the $R \$ \mathbf{5 , 0 0}$ note?", (b) Positive reinforcement of hit 


\subsubsection{Test case 2: quantification of banknote prices} Activity 2 teaches about the value of notes and how to make a simple purchase. The flowchart in Figure 3 was applied using the character Sara. Figure 7a shows the circuit sticker modeling of test case 2 on MangaSticker. Figure 7b shows the simulation of test case 2 .

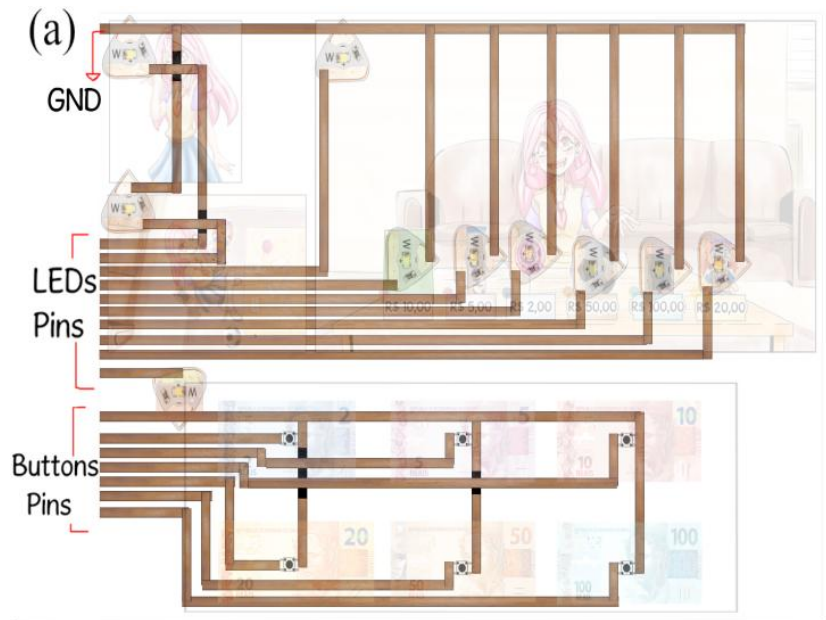

(b)

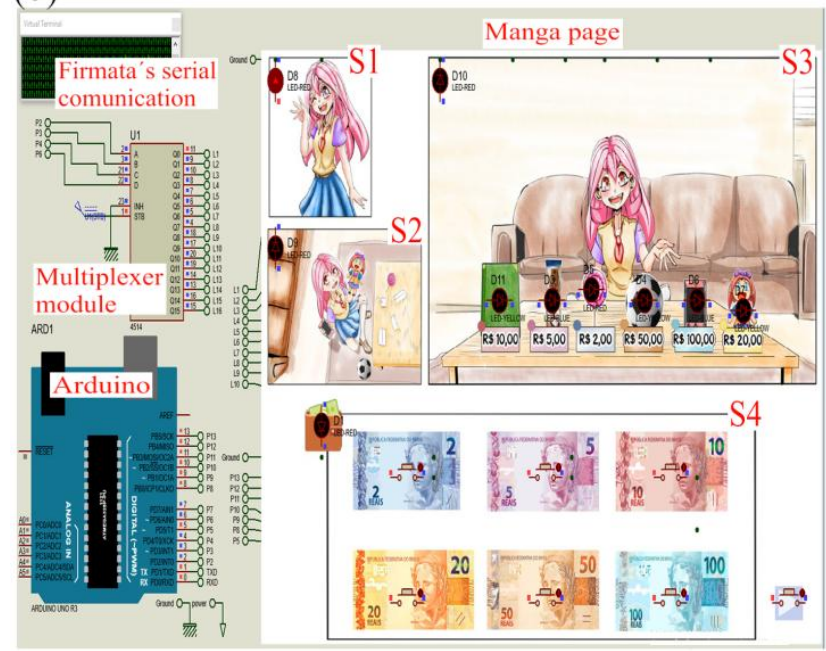

Fig 7: Test case 2: (a) MangaSticker screen, circuit sticker face (manga opacity reduced), (b) Simulation

The scenes were divided into four frames S1, S2, S3, and S4. Sara is a girl who likes to play shopping. In the beginning, Sara introduces herself to the interlocutor and invites him to her favorite game (shopping). During the game's explanation, the character shows how the money bills can be combined one by one with the objects. The "one-to-one" note-to-object relation reduces the use of pre-arithmetic skills, facilitating activity for people with DS.

After the explanation, Sara begins the activity by asking the interlocutor to buy the objects she indicates. The questions are answered by pressing the note chosen in frame S4 (wallet). Positive reinforcement is performed with each response. If it is a hit, audios and lighting effects are played for celebration. If it is an error, the character encourages a second attempt. In the end, the character congratulates the interlocutor and says goodbye with an invitation for the next time.

In the circuit sticker, the Arduino controls the LEDs that indicate frames and items in the scene accordingly with the character's speech. The buttons were positioned below the frame S4 notes (wallet), allowing interaction with the manga to answer questions.

\subsubsection{Test case 1 version 2: without a controller}

An interactive manga was created and simulated without the Arduino use. Figure 8 shows the circuit diagram mounted in the MangaSticker. The face of the manga remained the same, as shown in Figure 5a. Figure 9 shows the simulation of test case 1 , version 2 .

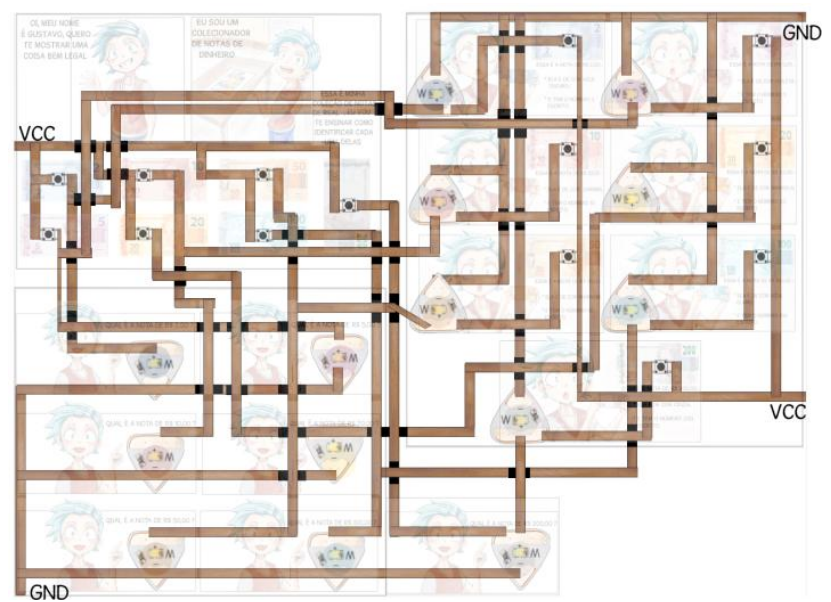

Fig 8: MangaSticker screen, test case 1 version 2, circuit sticker

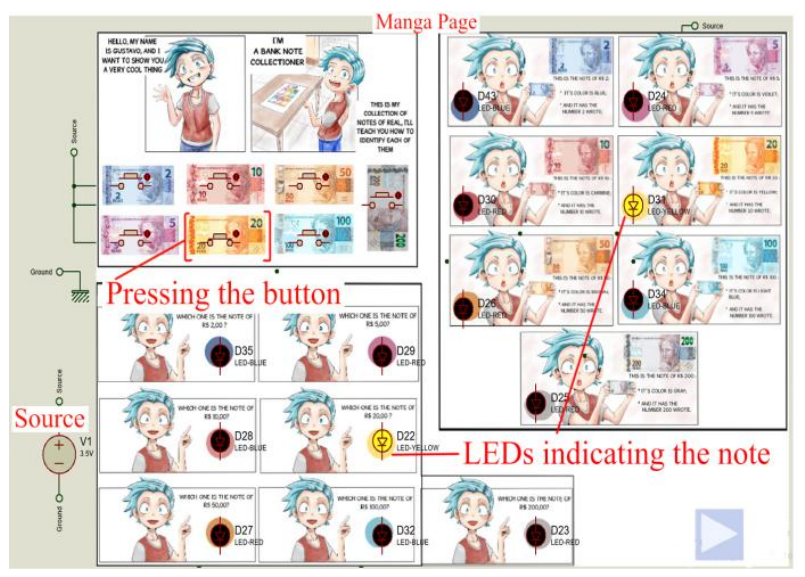

Fig 9: Simulation of test case 1 version 2, question: "Which one is the note of $\mathbf{R} \$ \mathbf{2 0 . 0 0 ? "}$

Unlike version 1 with Arduino, this version does not play audio. It makes it necessary to read all of the character's lines to follow the story. The positive reinforcement consists only of pre-positioned LEDs that indicate which question refers to the note being clicked. A 3.5v source powers the circuit. This version guarantees more portability and can be stored in catalog folders or binders. The assembly of the circuit is also simple, which shows that not much technical knowledge is required to assemble interactive manga.

\section{RESULTS AND DISCUSSIONS}

This paper proposes the development of MangaSticker, a tool to build interactive manga in the form of a circuit sticker. Figures 2 and 5 show that using an editing area with the manga and circuit sticker items superimposed facilitates interactive manga modeling. A virtual environment for circuit sticker production allows corrections to be made quickly and several times, which in general is not possible in commons circuit sticker projects.

The literature review verified that the circuit sticker kits generally do not include tools to generate figures or new 
circuit models virtually, being necessary to create them freehand on paper. Manual illustration skills are not mandatory in MangaSticker. All graphical components can be downloaded from the internet or chosen from the standard figures. Users can also model their sprites and add them to the software using the add figure function. It gives users more creative freedom, allowing the customization of artifacts according to their ideas and preferences.

Both the editing and printing of the project occur based on the dimensions of the A4 sheet. A4 sheets are familiar and inexpensive. It is possible to create a large-scale project, which can be explored in future test cases.

The evaluation of MangaSticker was done by implementing three test cases for training people with DS, shown in Section 3.2. The test cases show that it is possible to use interactive manga in teaching. They can be customized for training people with DS, which can also be done for other populations. The activities' modeling showed the MangaSticker's capacity to set the scenarios, position the characters, and model the circuit sticker.

Arduino and the Firmata protocol in implementing the test cases enabled the integration between the interactive manga and the computer. It was possible to control the scenes and assign lines to the characters, being a quick method due to the Firmata protocol's simplicity. In the physical implementation of test case 1, the tool's validation is verified when printing the models. Therefore, it is more practical to use the model generated by MangaSticker than to do it freehand, as is usually done in other circuit sticker projects.

A version of test case 1 was simulated without the presence of the controller. Such a version presented losses in the audio and in the control of the scenes. This case is not highly recommended for the population with DS. On the other hand, it has good portability and simpler circuits.

Two characters were generated, Gustavo and Sara, modeled based on the population's requirements (Down Syndrome), making the characters' reuse in other activities possible. More familiar characters names have also been added to the tool, which can be used in any interactive manga project.

\section{CONCLUSION}

This paper proposes developing a tool for building interactive manga in the form of a circuit sticker. It provides a simple way to create interactive artifacts that combine the manga's visual potential with the circuit sticker's interactivity. The tool allows the customization of interactive manga for different contexts, including teaching. The test cases showed that MangaSticker could be used to produce interactive manga for training people with DS. Skills training with money is essential for the autonomous life of individuals with DS. The concept of interactive manga through circuit stickers and educational manga has not been found in the literature. There were also not found studies using educational manga or circuit sticker for the population with DS.

It is understood as future works, testing the proposed activities with volunteers from the Alfadown project, which was impossible due to the covid-19 quarantine. Tests can contribute to the job, for example, data on attention and engagement in activities with interactive manga. New activities should be created for teaching Downs Syndrome people with credit card usage, which is a modern means of payment. A timeline must be added to the application, allowing several interactive manga pages in the same project. Firmata can be integrated into MangaStiker. It would make it possible to create animations and character controls on the computer screen through interaction with the interactive manga. Finally, options must be added to change the editing area's dimensions to explore artifacts in other sizes, such as A2, A3, or A6.

A version of the MangaSticker software is available free of charge at https://github.com/Vitor0534/MangaSticker.git

\section{ACKNOWLEDGMENTS}

Special thanks to the people of the Alfadown project, who supported this work.

\section{REFERENCES}

[1] Qi, J., Huang, A. "bunnie" and Paradiso, J. (2015). Crafting technology with circuit stickers. Proceedings of the 14th International Conference on Interaction Design and Children - IDC ' 15 .

[2] QI, Jie. Paper Electronics: "Circuits on Paper for Learning and Self-Expression”. 2016. 217 f. Tese (Doutorado) - Curso de Media Arts And Sciences, Massachusetts Institute Of Technology, Massachusetts Institute Of Technology, 2016.

[3] BERNDT, Dr. Jaqueline. "Manga meets Science: Going beyond the Education-Entertainment Divide". Berlin: Dfg, 2017.

[4] MURAKAMI, Satsuki. "Manga as an Educational Medium". The International Journal Of The Humanities, [S. L.], v. 7, n. 10, p. 47-55, 2009.

[5] Iwata, A. and Luppeti, K. (2018). UTILIZANDO A NARRATIVA SEQUENCIAL DOS MANGÁS PARA ILUSTRAR CONCEITOS DE QUÍMICA. Revista Debates em Ensino de Química, pp.51-72.

[6] PUC, Goiás. (2020) [Online]. Available in: http://sites.pucgoias.edu.br/extensao/coordenacoes/coord enacao-de-extensao/programa-de-referencia-em inclusaosocial/.

[7] PLIX. Scratch+micro:bit. 2020. Available in: https://plix.media.mit.edu/kits/scratch-microbit/.

[8] CHIBITRONICS. Project category: Craft. 2018. Available in: <https://chibitronics.com/projectcategory/craft/page/2/?wpv_view_count=6102>.

[9] Jacoby, S. and Buechley, L. (2013). "Drawing the electric". Proceedings of the 12th International Conference on Interaction Design and Children - IDC '13.

[10] SANTOS, Danilo Alves. Wearable Device for Literacy Activities with People with Down Syndrome. Goiânia: Puc Go, 2017.

[11] Peiris, R.L. and Nanayakkara, S. (2014). PaperPixels. Proceedings of the 26th Australian Computer-Human Interaction Conference on Designing Futures the Future of Design - OzCHI '14.

[12] Ngai, G., Chan, S.C.F., Ng, V.T.Y., Cheung, J.C.Y., Choy, S.S.S., Lau, W.W.Y. and Tse, J.T.P. (2010) $i *$ CATch. Proceedings of the 28th international conference on Human factors in computing systems CHI '10.

[13] Sylla, C., Branco, P., Gonçalves, S., Coutinho, C. and Brito, P. (2012). t-books. Proceedings of the 11th International Conference on Interaction Design and 
Children - IDC '12.

[14] Maddumarachchi, J. (2015). "Using Video Modelling and Video Self-Modelling to Teach Money Handling Skills to Adolescents and Young Adults with Down syndrome". PhD. p.100.

[15] Rus, S., Caliz, D., Braun, A., Engler, A. and Schulze, E. (2017). "Assistive apps for activities of daily living supporting persons with Down's Syndrome". Journal of Ambient Intelligence and Smart Environments, 9(5), pp.611-623.

[16] Fundação síndrome de down. o que é síndrome de down? 2019. Available in: http://www.fsdown.org.br/sobre-asindrome-de-down/o-que-e-sindrome-de-down/.

[17] Reas, Casey; FRY, Ben. Render Techniques. 2014. Available in: https://processing.org/tutorials/rendering/.
[18] Hong, Seungjin et al. Game Sprite Generator Using a Multi Discriminator GAN. Ksii Transactions On Internet And Information Systems, Hongik, v. 13, n. 8, p. 42554269, 8 ago. 2019.

[19] Sojamo. Package controlP5. 2016. Available in: http://www.sojamo.de/libraries/controlP5/reference/.

[20] Gonzales, Rafael, and Richard Woods. Processamento Digtal de Imagem. Vol. 3, São Paulo, SV, 2010.

[21] Protosupplies. 16-Ch Analog / Digital Mux Module. 2020. Available in: https://protosupplies.com/product/16ch-analog-digital-mux-module/.

[22] Firmata (org.). Main Page. 2018. Available in: http://firmata.org/wiki/Main_Page. 